\title{
A PAIR OF INDICES FOR FUNCTION SPACES ON THE CIRCLE
}

\author{
BY
}

\section{COLIN BENNETT}

\begin{abstract}
We give here some of the basic properties of the classes $\left\{\Phi_{r}\right\}$, $\left\{\Psi_{r}\right\},-1<r<1$, of dilation operators acting in rearrangement-invariant spaces $x$ on the circle It is shown that to each space $¥$ there correspond two numbers $\xi$, $\eta$, called indices, which satisfy $0 \leq \eta \leq \xi \leq 1$; the se numbers represent the rate of growth or decay of $\left\|\Psi_{r}\right\|$ as $r \rightarrow \pm 1$.

By using the operators $\stackrel{r}{\Psi}_{r}$ to obtain estimates for certain averaging operators $A_{\gamma}$, we are able to show that the indices $(\xi, \eta)$ coincide with the Boyd indices $(\alpha, \beta)$. As a consequence, we obtain a Marcinkiewicz-type interpolation theorem for rearrangement-invariant spaces on the circle.
\end{abstract}

1. Introduction. Using the device of mapping functions $f$ onto their nonincreasing rearrangements $f^{*}$, Luxemburg [12] has shown that the study of rearrangement-invariant spaces over arbitrary measure spaces reduces to that of rearrangement-invariant spaces defined on an interval or half-line. Thus most of the interpolation theorems for these spaces are framed in terms of the nonincreasing rearrangements $f^{*}$ (cf. [4], [6], [7], [9], [10], [14]). Typically, such a theorem would state that a rearrangement-invariant space satisfies some interpolation property if certain restrictions are met on the norms $\left\|E_{s}\right\|$ of the dilation operators $E_{s}$ (defined by $E_{s} f^{*}(t)=f^{*}(s t)$ ). For example, Boyd [6] gave necessary and suf ficient conditions on the indices $\alpha, \beta$ (the "rates of growth" of $\left\|E_{s}\right\|$ as $s \rightarrow$ 0 and $s \rightarrow \infty)$ in order that a Marcinkiewicz-type theorem be valid.

The main purpose of this paper is to characterize interpolation properties of rearrangement-invariant spaces $\mathfrak{X}$ on the circle in terms of growth conditions on certain operators which act directly on the functions in $\mathfrak{X}$ and not on their nonincreasing rearrangements; the operators involved are the dilation operators $\Phi_{r}$, $\Psi_{r}$ introduced by the author in [1]. This enables us to apply the operators $\Phi_{r}$ and $\Psi_{r}$ in other directions (for instance, to obtain estimates for integral averaging operators as in [1], [2], [3]) while at the same time knowing something of the interpolation properties of the space $\mathfrak{X}$. Sharper versions of theorems in [1] result but these will be announced elsewhere.

In $\$ 2$, we summarize some of the more important results on rearrangement-

Received by the editors November 8, 1971 .

A.MS (MOS) subject classifications (1970). Primary 46E30, 43A15; Secondary 46E 35.

Key words and phrases. Rearrangement-invariant space, interpolation theorem, dilation operator, indices. 
invariant spaces and $\$ \S 3,4$ are concerned with the basic properties of the operators $\Phi_{r}, \Psi_{r}$. Most of these results (in somewhat weaker form) appeared in [1] but the construction of the indices $(\S 3)$ and their characterization in terms of the operators $\Psi_{r}$ (Theorem 4.5 ) are new. It is shown in $\$ 5$ that the question of boundedness for the averaging operators $A_{\gamma}$ can be answered in terms of the indices $\xi, \eta$ (Theorem 5.6). It is then fairly easy to see that the indices coincide with the Boyd indices $\alpha, \beta$ (Theorem 6.2). As a result, we obtain an interpolation theorem (Theorem 6.3) for weak type operators acting on rearrangement-invariant spaces on $T$.

2. Rearrangement-invariant spaces. Let $T$ be the unit circle $T=\left\{e^{i \theta}\right.$ : $0 \leq \theta<2 \pi\}$ with Lebesgue measure $d t$; we shall frequently write $d m=(1 / 2 \pi) d t$ so that $m(T)=1$. $\mathbb{N}$ and $\mathcal{P}$ denote respectively the classes of $m$-measurable and nonnegative $m$-measurable functions on $T$. The symbol $\chi_{E}$ will be reserved for the characteristic function of a subset $E$ of $T$.

The following notions of function norm, etc., are due to Luxemburg ([11], [12]); see also [4], [6]. A function norm $\mu: \mathcal{P} \rightarrow[0, \infty]$ is a mapping which sat isfies the following conditions for all $f, g, f n$ in $\mathcal{P}$ and all constants $\lambda \geq 0$ :

(2.1) $\mu(f)=0 \Leftrightarrow f=0$ a.e.,

(2.2) $\mu(\lambda f)=\lambda \mu(f)$,

(2.3) $\mu(f+g) \leq \mu(f)+\mu(g)$,

(2.4) $f \leq g$ a.e. $\Rightarrow \mu(f) \leq \mu(g)$,

(2.5) $\mu(1)=1$,

(2.6) $(1 / 2 \pi) \int_{0}^{2 \pi} f(t) d t=\int_{T} f d m \leq$ const $\mu(f)$,

(2.7) fn $\uparrow$ a.e. $\Rightarrow \mu(f n) \uparrow \mu(f)$.

When functions differing only on a set of measure zero are identified, the set $\mathfrak{X}=L^{\mu}$, consisting of all functions $f \in \mathbb{M}$ for which $\mu(|f|)<\infty$, is a Banach space under the norm $\|f\|_{\mathfrak{X}}=\mu(|f|), f \in \mathfrak{X}$. Such a space $\mathfrak{X}$ is called a Banach function space; it is immediate from the definition (2.1), ., (2.7) that $L^{\infty} \subset X \subset L^{1}$ (and each inclusion is continuous).

We define the distribution function $D_{f}:[0, \infty) \rightarrow[0,1]$ of a measurable function $f$ by

$$
D_{f}(y)=m\{\theta:|f(\theta)|>y\}, \quad 0 \leq y<\infty
$$

(where we have written $f(\theta)$ instead of $f\left(e^{i \theta}\right)$ to economize on notation). The nonincreasing rearrangement $f^{*}$ of $f \in L^{1}$ is the function $f^{*}:[0,1] \rightarrow[0, \infty]$ defined by

$$
f^{*}(t)=\inf \left\{y: D_{f}(y) \leq t\right\}, \quad 0 \leq t \leq 1 .
$$

Two functions $f$ and $g$ with $D_{f}=D_{g}$ are called equimeasurable and we write $f \sim g$. A Banach function space is rearrangement-invariant if, along with $f$, it 
contains every function on $T$ equimeasurable with $f$; by an equivalent renorming we may (and shall) assume that the norm in a rearrangement-invariant space $\mathfrak{X}$ satisfies (cf. [12])

$$
f \sim g \Rightarrow\|f\|_{\mathfrak{X}}=\|g\|_{\mathfrak{X}}, \quad f, g \in \mathfrak{X}
$$

In particular, translation in $X$ is an isometry.

A function $f$ on $T$ will be called bell-shaped if it is nonnegative, nonincreasing on $[0, \pi]$ and $f(-\theta)=f(\theta), 0 \leq \theta \leq \pi$. The bell-shaped rearrangement of $f$ is, by definition, the bell-shaped function $f^{\#}$ equal to $f^{*}(\theta / \pi)$ on $[0, \pi]$. It is clear that $f^{\#}$ is equimeasurable with both $f$ and $f^{*}$; hence $\left\|f^{\#}\right\|_{x}=\|f\|_{x}$.

The associate space $\mathfrak{X}^{\prime}$ of $\mathfrak{X}$ is again a rearrangement-invariant space; its norm is defined by

$$
\|g\|_{X^{\prime}}=\sup \left\{(f, g\rangle:\|f\|_{X} \leq 1\right\}, \quad g \in X^{\prime},
$$

where $\langle f, g\rangle=\int f g d m$.

Lorentz (unpublished) and Luxemburg ([11], [12]) showed independently that $\mathfrak{X}^{\prime \prime}=\left(X^{\prime}\right)^{\prime}=\mathfrak{X}$ so that it follows from (2.9) that

$$
\|f\|_{X}=\sup \left\{\langle f, g\rangle:\|g\|_{x^{\prime}} \leq 1\right\}, \quad f \in \mathfrak{X} .
$$

In terms of bell-shaped rearrangements, we have (cf. [12, Theorem 11.9])

$$
\begin{aligned}
& \|g\|_{X^{\prime}}=\sup \left\{\left\langle f^{\#}, g^{\#}\right\rangle:\|f\|_{\mathfrak{X}} \leq 1\right\}, \quad g \in \mathfrak{X}^{\prime}, \\
& \|f\|_{\mathfrak{X}}=\sup \left\{\left\langle f^{\#}, g^{\#}\right\rangle:\|g\|_{\mathfrak{X}^{\prime}} \leq 1\right\}, \quad f \in X .
\end{aligned}
$$

One consequence of the expressions (2.9), $\cdots,(2.12)$ is the Hölder inequality

$$
\langle|f|,|g|\rangle \leq\left\langle f^{\#}, g^{\#}\right\rangle \leq\|f\|_{\mathfrak{X}}\|g\|_{\mathfrak{X}^{\prime}}, \quad f \in \mathfrak{X}, g \in \mathfrak{X}^{\prime},
$$

which we shall use frequently.

We conclude this section by stating Calderón's interpolation theorem [7]. Recall that an operator $S$ is of strong type $(p, q)$ if $S$ maps $L^{p}$ continuously into $L^{q}$.

Theorem 2.1 (Calderón). Let $\mathfrak{X}$ be a rearrangement-invariant space on the circle. If $S$ is a linear operator of strong types $(1,1)$ and $(\infty, \infty)$ (with norms not exceeding 1) then $S$ maps $X$ continuously into itself (with norm not exceeding 1 ).

Corollary 2.2. (a) $X$ is a module over $L^{1}$ via convolution and $\|f * g\|_{X} \leq$ $\|f\|_{\mathfrak{X}}\|g\|_{1}, f \in \mathfrak{X}, g \in L^{1}$.

(b) $X$ is a module over $L^{\infty}$ via pointwise multiplication and $\|f g\|_{\mathfrak{X}} \leq$ $\|f\|_{\mathfrak{X}}\|g\|_{\infty}, f \in \mathfrak{X}, g \in L^{\infty}$. 
3. The operators $\Phi_{r}$. For each $r,-1<r<1$, let $\phi_{r}$ be the linear fractional transformation

$$
\phi_{r}\left(e^{i \theta}\right)=\left(e^{i \theta}-r\right) /\left(1-r e^{i \theta}\right), \quad e^{i \theta} \in T .
$$

Each mapping $\phi_{r}$ is a continuous, one-to-one mapping of $T$ onto itself leaving -1 and 1 fixed. Under the operation of composition, the mappings $\phi_{r}$ form a group, the identity being the identity mapping $\phi_{0}$ and the inverse of $\phi_{r}$ being $\phi_{-r}$. Consequently, each operator $\Phi_{r}$ defined by

$$
\left(\Phi_{r} f\right)\left(e^{i \theta}\right)=\left(f \circ \phi_{r}\right)\left(e^{i \theta}\right)=f\left(\phi_{r}\left(e^{i \theta}\right)\right), \quad e^{i \theta} \in T,
$$

is an automorphism of the measurable functions $M$. When $e^{i \theta} \neq \pm 1$, we see from (3.1) that

$$
\lim _{r \rightarrow-1} \phi_{r}\left(e^{i \theta}\right)=1, \quad \lim _{r \rightarrow 1} \phi_{r}\left(e^{i \theta}\right)=-1,
$$

so if $f$ is continuous and $e^{i \theta} \neq \pm 1$, we have

$$
\lim _{r \rightarrow-1}\left(\Phi_{r} f\right)\left(e^{i \theta}\right)=f(1), \quad \lim _{r \rightarrow 1}\left(\Phi_{r} f\right)\left(e^{i \theta}\right)=f(-1) .
$$

This last property exhibits the "dilation" behavior of $\Phi_{r}$.

The usual dilation operators $E_{s}, 0<s<\infty$, for the line are defined by

$$
\left(E_{s} f\right)(t)=f(s t), \quad-\infty<t<\infty \text {. }
$$

Since, under the usual mapping $e^{i \theta} \rightarrow i\left(1-e^{i \theta}\right) /\left(1+e^{i \theta}\right)$ of the circle onto the (extended) real line, $\Phi_{r}$ corresponds to $E_{s}, s=(1+r) /(1-r)$, it is perhaps not surprising that $\Phi_{r}$ can be used in many situations for the circle where $E_{s}$ would be used for the line.

In the next proposition we list without proof some simple properties of the operators $\Phi_{r}$. For each $r,-1<r<1, P_{r}$ will denote the Poisson kernel

$$
P_{r}(\theta)=\left(1-r^{2}\right) /\left(1-2 r \cos \theta+r^{2}\right), \quad e^{i \theta} \in T
$$

Proposition 3.1. (a) The operators $\Phi_{r}$ form a group and

$$
\Phi_{r} \Phi_{s}=\Phi_{t}, \quad t=(r+s) /(1+r s), \quad-1<r, s<1 .
$$

(b) For each $r,-1<r<1$, the change of variable $\phi_{r}\left(e^{i \theta}\right)=e^{i t}$ gives

$$
\frac{1}{2 \pi} \int_{0}^{2 \pi}\left(\Phi_{r} f\right)(\theta) d \theta=\frac{1}{2 \pi} \int_{0}^{2 \pi} f(t) P_{-r}(t) d t, \quad f \in \mathbb{M} \text {. }
$$

(c) For each $r,-1<r<1$,

$$
\Phi_{r}(1+\cos \theta)=((1-r) /(1+r))(1+\cos \theta) P_{r}(\theta), \quad e^{i \theta} \in T .
$$


Our next result shows, roughly speaking, that the "amount of stretching" effected by $\Phi_{r},-1<r<0$, is greatest near the point $e^{i \theta}=1$.

Proposition 3.2. For every integrable function $f$ on $T$ and $-1<r<0$,

$$
\left(\Phi_{r} f\right)^{\#} \leq \Phi_{r}\left(f^{\#}\right)
$$

(where $f^{\#}$ is the bell-shaped rearrangement of $f$ as in $\$ 2$ ).

Proof. First, let us establish (3.7) when $f$ is the characteristic function $\chi$ of some measurable subset $E$ of $T$. Denote by $E^{\#}$ the set of points where $\chi^{\#}$ $>0$. Clearly $m(E)=m\left(E^{\#}\right)$. Now using a change of variable as in (3.5) we have

$$
\begin{aligned}
m\left\{\theta:\left(\Phi_{r} \chi\right)(\theta)>0\right\} & =\int_{T}\left(\Phi_{r} \chi\right)(\theta) d m(\theta)=\int_{T} \chi\left(\phi_{r}\left(e^{i \theta}\right)\right) d m(\theta) \\
& =\int_{T} \chi(t) P_{-r}(t) d m(t)=\int_{E} P_{-r}(t) d t \\
& \leq \int_{E}^{\#} P_{-r}(t) d t=m\left\{\theta: \Phi_{r}\left(\chi^{\#}\right)(\theta)>0\right\} .
\end{aligned}
$$

It follows that $\left(\Phi_{r} \chi\right)^{\#} \leq \Phi_{r}\left(\chi^{\#}\right)$.

If now $f$ is a nonnegative simple function on $T$ then [12, Lemma 8.1] $f$ can be written in the form $f=\sum_{k=1}^{n} C_{k} X_{k}$ where $C_{k}>0, \chi_{k}$ is the characteristic function of a measurable set $E_{k}$ and $E_{1} \supset E_{2} \supset \ldots \supset E_{n}$. As a result we have $f^{\#}=\Sigma C_{k} \chi_{k}^{\#}$. But $\Phi_{r}$ is linear and so $\Phi_{r} f=\Sigma C_{k} \Phi_{r} \chi_{k}$; since $\Phi_{r} \chi_{1} \geq \Phi_{r} \chi_{2} \geq$ $\cdots \geq \Phi_{r} \chi_{n}$ it follows again from [12, Lemma 8.1] that $\left(\Phi_{r} f\right)^{\#}=\Sigma C_{k}\left(\Phi_{r} \chi_{k}\right)^{\#}$. Combining all these facts and using the first part of the proof we deduce that

$$
\left(\Phi_{r} f\right)^{\#}=\sum C_{k}\left(\Phi_{r} \chi_{k}\right)^{\#} \leq \sum C_{k} \Phi_{r}\left(\chi_{k}\right)^{\#}=\Phi_{r}\left(\sum C_{k} \chi_{k}^{\#}\right)=\Phi_{r}\left(f^{\#}\right) \text {. }
$$

This establishes (3.7) for $f$ simple and nonnegative.

When $f$ is an arbitrary nonnegative integrable function on $T$, there is a sequence of nonnegative simple functions $f_{n}$ on $T$ such that $f_{n} \uparrow f$ a.e. It is not hard to see (via the montone convergence theorem, for instance) that $f_{n}^{\#} \uparrow f^{\#}$ and hence that $\Phi_{r}\left(f_{n}^{\#}\right) \uparrow \Phi_{r}\left(f^{\#}\right)$. In exactly the same way, $\left\{\Phi_{r} f_{n}\right\}$ is a sequence of simple functions converging a.e. to $\Phi_{r} f$ and so $\left(\Phi_{r} f_{n}\right)^{\#} \uparrow\left(\Phi_{r} f\right)^{\#}$ a.e. Hence by (3.8),

$$
\left(\Phi_{r} f\right)^{\#}=\lim _{n \rightarrow \infty}\left(\Phi_{r} f_{n}\right)^{\#} \leq \lim _{n \rightarrow \infty} \Phi_{r}\left(f_{n}^{\#}\right)=\Phi_{r}\left(f^{\#}\right) .
$$

Finally the restriction that $f$ be nonnegative is removed by observing that $f^{\#}=$ $|f|^{\#}$.

Now let us consider the action of $\Phi_{r}$ on various rearrangement-invariant spaces. When $\Phi_{r}$ is a bounded operator from a Banach space $X$ into itself we 
shall denote by $\sigma_{x}(r)$ the operator norm of $\Phi_{r}$; when $\mathfrak{X}=L^{p}, 1 \leq p \leq \infty$, we shall write $\sigma_{p}(r)$ for $\sigma_{L p}(r)$.

Proposition 3.3. (a) $\Phi_{r}$ is an automorphism of $L^{1}$ and

$$
\sigma_{1}(r)=(1+|r|) /(1-|r|), \quad-1<r<1 .
$$

(b) $\Phi_{r}$ is an (isometric) automorphism of $L^{\infty}$ and

$$
\sigma_{\infty}(r)=1, \quad-1<r<1 .
$$

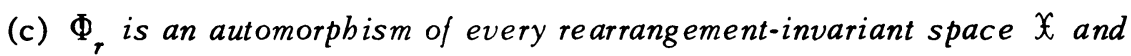

$$
\sigma_{\mathfrak{x}}(r) \leq(1+|r|) /(1-|r|), \quad-1<r<1 .
$$

Proof. (a) Using (3.5) we have

$$
\begin{aligned}
\sigma_{1}(r) & =\sup \left\{\left\|\Phi_{r} f\right\|_{1}:\|f\|_{1} \leq 1\right\}=\sup \left\{\int \Phi_{r}(|f|) d m:\|f\|_{1} \leq 1\right\} \\
& =\sup \left\{\int|f| P_{-r} d m:\|f\|_{1} \leq 1\right\}=\left\|P_{-r}\right\|_{\infty}=(1+|r|) /(1-|r|) .
\end{aligned}
$$

(b) The second part is obvious in view of the definition (3.2) of $\Phi_{r}$.

(c) The third part follows from parts (a) and (b) and Theorem 2.1.

Theorem 3.4. For each rearrangement-invariant space $\mathfrak{X}$, the limit

$$
\xi=\xi(X)=\lim _{r \rightarrow 1} \frac{\log \sigma_{\mathfrak{X}}(r)}{\log ((1+r) /(1-r))}
$$

exists and satisfies

$$
0 \leq \xi \leq 1
$$

Proof. If $\Phi_{t}^{\prime}=\Phi_{\tanh t},-\infty<t<\infty$, then (3.4) can be rewritten in the form $\Phi_{s+t}^{\prime}=\Phi_{s}^{\prime} \Phi_{t}^{\prime},-\infty<s, t<\infty$. Writing $\sigma_{x}^{\prime}(t)$ for the operator norm of $\Phi_{t}^{\prime}$, we deduce that the function $g(t)=\log \sigma_{\mathfrak{X}}^{\prime}(t)$ is subadditive and hence [8, p. 244] that the limit $\xi=\lim _{t \rightarrow \infty} g(t) / 2 t$ exists. The change of variable $r=\tanh t$ now produces (3.12).

That $\xi$ does not exceed 1 is immediate from (3.11) and (3.12). Furthermore, it is easy to see that $\Phi_{r}(1)=1$ implies $\sigma_{x}(r) \geq 1$. It follows that $\xi \geq 0$ and the proof is complete.

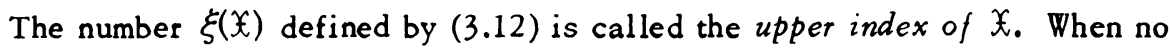
confusion can arise we shall write $\xi^{\prime}$ instead of $\xi\left(X^{\prime}\right)$, where $X^{\prime}$ is the

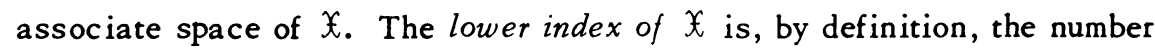

$$
\eta=1-\xi^{\prime}
$$

It is clear that

$$
0 \leq \eta, \xi \leq 1
$$


in fact, we shall prove in $\$ 4$ that $\eta \leq \xi$, hence justifying our terminology.

4. The operators $\Psi_{r}$. We remarked in $\$ 3$ that there is a natural correspondence betwe en the operators $\Phi_{r}$ on the circle and the operators $E_{s}$ on the line. How ever, many of the functions occuring in harmonic analysis vanish (in some sense) at infinity, whereas, on the circle, functions will not in general vanish at the point $e^{i \theta}=-1$. Consequently, the operators $\Psi_{r}$ defined by

$$
\Psi_{r} f=\Phi_{r}[1 / 2(1+\cos ) \cdot f],(1) \quad-1<r<1,
$$

are more important in the applications than the $\Phi_{r}$ (cf. [1], [2], [3]); the factor $1 / 2(1+\cos )$ is built in simply to provide $f$ with a "zero" at the point $e^{i \theta}=-1$.

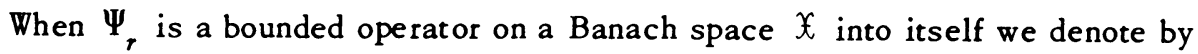
$\rho_{x}(r)$ the operator norm of $\Psi_{r}$; here again we write $\rho_{p}(r)$ for $\rho_{L}{ }^{(r)}$.

Our main objective (Theorem 4.5) in this section is to show that the indices $\xi, \eta$ can be defined in terms of $\rho_{\mathfrak{X}}(r)$ without reference to the associate space.

Proposition 4.1. (a) $\Psi_{r}$ is a bounded operator on $L^{1}$ and

$$
\rho_{1}(r)=(1-r) /(1+r), \quad-1<r<1 \text {. }
$$

(b) $\Psi_{r}$ is a bounded operator on $L^{\infty}$ and

$$
\rho_{\infty}(r)=1, \quad-1<r<1 .
$$

(c) $\Psi_{r}$ is a bounded operator on any rearrangement-invariant space $X$ and

$$
\rho_{x}(r) \leq \max (1,(1-r) /(1+r)), \quad-1<r<1 .
$$
$\left(L^{\infty}\right)^{\prime}$.

Proof. Part (a) follows from (b), the next proposition and the fact that $L^{1}=$

To prove (b), observe that

$\rho_{\infty}(r)=\sup \left\{\left\|\Psi_{r} f\right\|_{\infty}:\|f\|_{\infty} \leq 1\right\}=\sup \left\{\left\|\Phi_{r}[1 / 2(1+\cos ) \cdot f]\right\|_{\infty}:\|f\|_{\infty} \leq 1\right\}$.

But $\Phi_{r}$ is an isometry on $L^{\infty}$ (Proposition $3.4(\mathrm{~b})$ ) so

$$
\rho_{\infty}(r)=\sup \left\{\|1 / 2(1+\cos ) \cdot f\|_{\infty}:\|f\|_{\infty} \leq 1\right\}=1 .
$$

Part (c) follows from (a), (b) and Theorem 2.1. related.

Our next result shows that the operators $\Psi_{r}$ on $X$ and on $X^{\prime}$ are intimately

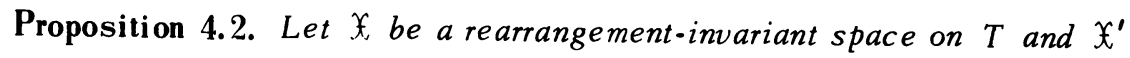
its associate space. Then

$$
\rho_{X}(r)=((1-r) /(1+r)) \rho_{x^{\prime}}(-r), \quad-1<r<1 .
$$

(1) $(1+\cos ) \cdot f$ denotes the function $t \rightarrow(1+\cos t) f(t)$. 
Proof. From (2.10), for any $f \in \mathfrak{X}$,

$$
\left\|\Psi_{r} f\right\|_{X}=\sup \left\{\left\langle\Psi_{r} f, g\right\rangle:\|g\|_{X^{\prime}} \leq 1\right\} .
$$

But using successively (4.1), (3.6) and (3.5), we deduce that

$$
\begin{aligned}
\left\langle\Psi_{r} f, g\right\rangle & =\left\langle\left(\Phi_{r} f\right) 1 / 2 \Phi_{r}(1+\cos ), g\right\rangle \\
& =((1-r) /(1+r))\left\langle P_{r} \Phi_{r} f, 1 / 2(1+\cos ) \cdot g\right\rangle=((1-r) /(1+r))\left\langle f, \Psi_{-r} g\right\rangle
\end{aligned}
$$

and hence, from (2.13), that

$$
\left\langle\Psi_{r}|f|,|g|\right\rangle \leq((1-r) /(1+r)) \rho_{\mathfrak{X}^{\prime}}(-r)\|f\|_{X}\|g\|_{X^{\prime}} .
$$

Thus, (4.6) and (4.7) together show that

$$
\left\|\Psi_{r} f\right\|_{\mathfrak{X}} \leq((1-r) /(1+r)) \rho_{\mathfrak{X}^{\prime}}(-r)\|f\|_{\mathfrak{X}}, \quad f \in \mathfrak{X},
$$

which, in turn, proves that

$$
\rho_{\mathfrak{X}}(r) \leq((1-r) /(1+r)) \rho_{\mathfrak{X}}{ }^{\prime}(-r), \quad-1<r<1 .
$$

Replacing $X$ by $X^{\prime}$ and $r$ by $-r$ in (4.8) we find that $\rho_{x^{\prime}}(-r) \leq((1+r) /(1-r))$ - $\rho_{x}(r)$, and this estimate, together with (4.8), yields the desired result (4.5). The proof of Proposition 4.2 (and hence of 4.1 (a)) is now complete.

Our next two results illustrate the effect of the factor $1 / 2(1+\cos )$ occurring in the definition (4.1) of $\Psi_{r}$ : When $0 \leq r<1, \Psi_{r}$ is always a contraction but, when $-1<r<0, \Psi_{r}$ has the same "rate of growth" as ${ }_{r}$.

Proposition 4.3. When $0 \leq r<1$, we have

$$
\rho_{\mathfrak{X}}(r) \leq 1 \leq \rho_{\mathfrak{X}}(-r)
$$

and, moreover,

$$
\rho_{\mathfrak{x}}(r) \rho_{\mathfrak{x}}(-r) \geq 1
$$

Proof. The first inequality in (4.9) follows directly from (4.4). To establish the second half, fix $r$ with $0 \leq r<1$ and let $\epsilon>0$ be arbitrary. There exists $\delta=\delta(\epsilon)>0$ such that $1 / 2(1+\cos ) \cdot \chi_{\delta}>(1-\epsilon) \chi_{\delta}$ where $\chi_{\delta}$ is the characterist ic function of the set $E_{\delta}=\left\{e^{i \theta}:|\theta|<\delta\right\}$. Since $\Phi_{-r}$ "stretches" the set $E_{\delta}$ when $r>0$, it follows that

$$
\Psi_{-r} \chi_{\delta} \geq(1-\epsilon) \Phi_{-r} X_{\delta} \geq(1-\epsilon) \chi_{\delta}
$$

and hence that $\left\|\Psi_{-r} \chi_{\delta}\right\|_{\mathfrak{X}} \geq(1-\epsilon)\left\|_{\chi}\right\|_{\mathfrak{X}}$. But this implies that $\rho_{\mathfrak{X}}(-r) \geq 1-\epsilon$. We now use the fact that $\epsilon>0$ is arbitrary and (4.9) is established.

A similar argument is used to prove (4.10). Observe that

$$
\Psi_{r}\left[\Psi_{-r} f\right]=1 / 2(1+\cos ) \cdot f \Phi_{r}(1 / 2(1+\cos )) .
$$


But $1 / 2(1+\cos ) \cdot \Phi_{r}(1 / 2(1+\cos ))$ is continuous and equal to 1 when $\theta=0$. Hence, in the same way as before, we can find, for each $\epsilon>0$, a characteristic function $\chi$ such that $\left\|\Psi_{r}\left[\Psi_{-r} \chi\right]\right\|_{\mathfrak{X}} \geq(1-\epsilon)\left\|_{\chi}\right\|_{\mathfrak{X}}$. This implies that the operator norm $\left\|\Psi_{r} \Psi_{-r}\right\|$ is not less than 1 and hence that $\rho_{\mathfrak{X}}(r) \rho_{\mathfrak{X}}(-r) \geq\left\|\Psi_{r} \Psi_{-r}\right\| \geq 1$, as required.

Proposition 4.4. For each $r, 0 \leq r<1$,

$$
\rho_{\mathfrak{X}}(-r) \leq \sigma_{\mathfrak{x}}(-r) \leq 4 \rho_{\mathfrak{X}}(-r) .
$$

Proof. The first half of (4.11) is obvious in view of the definition (4.1) of $\Psi_{-r}$. To establish the second inequality we need to consider the characteristic functions $\chi_{E}$ of the set $\left\{e^{i \theta}:|\theta| \leq \pi / 2\right\}$ and $\chi_{F}$ of the set $F=T \backslash E$.

For any function $f \in X$ it is clear that $|f| \chi_{E} \leq(1+\cos ) \cdot|f| \chi_{E}$. Hence

$$
\left\|\Phi_{-r}\left(f \chi_{E}\right)\right\|_{\mathfrak{X}} \leq 2\left\|\Psi_{-r}\left(f \chi_{E}\right)\right\|_{\mathfrak{X}} \leq 2 \rho_{\mathfrak{X}}(-r)\|f\|_{\mathfrak{X}}, \quad-1<r<1 .
$$

On the other hand, if $S=S(\pi)$ is the operation of translation through $e^{i \pi}$, it is easy to see that $\Phi_{-r}=S \Phi_{r} S$. But then

$$
\Phi_{-r}\left(f \chi_{F}\right)=\left(S \Phi_{r} S\right)\left(f \chi_{F}\right)=S \Phi_{r}\left[(S f) \chi_{E}\right]
$$

so by (4.12)

$$
\left\|\Phi_{-r}\left(f \chi_{F}\right)\right\|_{\mathfrak{X}}=\left\|\left(S \Phi_{r}\right)\left[(S f) \chi_{E}\right]\right\|_{\mathfrak{X}}=\left\|\Phi_{r}\left[(S f) \chi_{E}\right]\right\|_{\mathfrak{X}} \leq 2 \rho_{\mathfrak{X}}(r)\|f\|_{\mathfrak{X}},
$$

because $S$ is an isometry on $\mathfrak{X}$.

Now if $f$ is any function in $X$ and $0 \leq r<1$ we can use (4.12), (4.13) and (4.9) to deduce that

$$
\begin{aligned}
\left\|\Phi_{-r} f\right\|_{\mathfrak{X}} & =\left\|\Phi_{-r}\left(f \chi_{E}+f \chi_{F}\right)\right\|_{\mathfrak{X}} \leq\left\|\Phi_{-r}\left(f \chi_{E}\right)\right\|_{\mathfrak{X}}+\left\|\Phi_{-r}\left(f \chi_{F}\right)\right\|_{\mathfrak{X}} \\
& \leq 2\left(\rho_{\mathfrak{X}}(r)+\rho_{\mathfrak{X}}(-r)\right)\|f\|_{\mathfrak{X}} \leq 4 \rho_{\mathfrak{X}}(-r)\|f\|_{\mathfrak{X}}
\end{aligned}
$$

and hence that $\sigma_{\mathfrak{X}}(-r) \leq 4 \rho_{\mathfrak{X}}(-r), 0 \leq r<1$. This completes the proof.

It would be of interest to know whether, in fact, $\rho_{\mathfrak{X}}(-r)$ is equal to $\sigma_{\mathfrak{X}}(-r), 0 \leq r<1$.

Theorem 4.5. Let $£$ be a rearrangement-invariant space on $T$ with indices $\xi, \eta$ defined as in $\$ 3$. Then

$$
\xi=\lim _{r \rightarrow 1} \frac{\log \rho_{x}(-r)}{\log ((1+r) /(1-r))}
$$

and

$$
\eta=\lim _{r \rightarrow 1} \frac{\log \rho_{x}(r)}{\log ((1-r) /(1+r))}
$$


Moreover, the indices satisfy

$$
0 \leq \eta \leq \xi \leq 1
$$

and

$$
\xi^{\prime}=1-\eta, \quad \eta^{\prime}=1-\xi .
$$

Proof. The equation (4.14) follows from (3.12) and (4.11), and (4.15) is immediate from (3.14), (4.5) and (4.14). In view of (3.15), to establish (4.16) we need only show that $\eta \leq \xi$; this follows directly from (4.10). Finally, the equalities (4.17) are consequences of the definition (3.14).

5. The averaging operators $A_{\gamma}$. For each $\gamma, 0 \leq \gamma \leq 1$, set

$$
\left(A_{\gamma} f\right)(t)= \begin{cases}(\tan t / 2)^{-\gamma} \int_{0}^{t}(\tan s / 2)^{\gamma-1} f(s) d s, & 0<t<\pi / 2 \\ 0, \quad \pi / 2 \leq t \leq 2 \pi\end{cases}
$$

$A_{\gamma} f$, extended by periodicty, is a function on $T$ and we shall be interested in estimating the norm of the operator $A_{\gamma}: f \rightarrow A_{\gamma} f$.

Theorem 5.1. Let $\mathfrak{X}$ be a rearrangement-invariant space on $T$ with upper index $\xi$. If $\xi<\gamma \leq 1$, then $A_{\gamma}$ is a bounded linear operator from $\mathfrak{X}$ into itself.

Proof. When $0<t<\pi / 2$, we have from (5.1)

$$
\left(A_{\gamma} f\right)(t)=(\tan t / 2)^{-\gamma} \int_{0}^{t}(\tan \theta / 2)^{\gamma-1} f(\theta) d \theta .
$$

Keeping $t$ fixed, we make the change of variable $e^{i t}=\phi_{r}\left(e^{i \theta}\right), 0<r<1$; it is not difficult to check that

$$
d \theta=\left(4 /(1+r)^{2}\right) \tan (t / 2) \cdot \Psi_{-r}(1) d r
$$

and that

$$
\Phi_{r}(\tan t / 2)=((1+r) /(1-r)) \tan t / 2
$$

Thus

$$
\left(A_{\gamma} f\right)(t)=\int_{0}^{1} \frac{4}{(1+r)^{2}}\left(\frac{1-r}{1+r}\right)^{\gamma-1}\left(\Psi_{-r} f\right)(t) d r, \quad 0<t<\pi / 2
$$

and so

$$
\left\|A_{\gamma} f\right\|_{x} \leq\left(4 \int_{0}^{1}(1-r)^{\gamma-1} \rho_{\mathfrak{x}}(-r) d r\right)\|f\|_{x} .
$$

Hence, to prove the theorem, we need only show that

$$
\int_{0}^{1}(1-r)^{\gamma-1} \rho_{\mathfrak{X}}(-r) d r<\infty
$$

Now $\xi<\gamma$ by hypothesis and so, for some sufficiently small $\epsilon>0$, we also 
have $\xi+\epsilon<\gamma$. But in view of (4.14), there exists $R=R(\epsilon), 0<R<1$, such that whenever $R<r<1$,

$$
\log \rho_{\mathfrak{X}}(-r) \leq(\xi+\epsilon) \log ((1+r) /(1-r)),
$$

i.e. $\rho_{x}(-r) \leq((1+r) /(1-r))^{\xi+\epsilon} \leq 2^{\xi+\epsilon}(1-r)^{-(\xi+\epsilon)} \leq 2(1-r)^{-(\xi+\epsilon)}$. Consequently,

$$
\int_{R}^{1}(1-r)^{\gamma-1} \rho_{\mathfrak{X}}(-r) d r \leq 2 \int_{R}^{1}(1-r)^{\gamma-(\xi+\epsilon)-1} d r<\infty
$$

because $\xi+\epsilon<\gamma$. Since the integrand is bounded over $[0, R]$, this establishes (5.5) and hence completes the proof.

Our immediate aim (Theorem 5.6) is to establish the converse of The orem 5.1 and this will be achieved in our next series of propositions. The proofs are, apart from a greater degree of technical difficulty, essentially those of Boyd ([4], [5], [6]). But first we need a lemma.

Lemma 5.2. For each $r, 0 \leq r<1$,

$$
\rho_{\mathfrak{X}}(-r)=\sup _{\|f\|_{\mathfrak{X}} \leq 1} \sup _{\boldsymbol{g} \|_{\mathfrak{X}^{\prime} \leq 1}}\left\langle\Psi_{-r}\left(f^{\#}\right), g^{\#}\right\rangle \text {. }
$$

Proof. The function $(1+\cos )$ is bell-shaped so, if $0<t<\pi$, it follows from $[12$, p. 110$]$ that

$$
\int_{0}^{t}[(1+\cos ) \cdot f]^{\#} d s \leq \int_{0}^{t}(1+\cos )^{\#} f^{\#} d s=\int_{0}^{t}(1+\cos ) \cdot f^{\#} d s, \quad f \in \mathfrak{X} .
$$

But, if $g \in X^{\prime}$, then $\Phi_{r}\left(g^{\#}\right) P_{r}$ is decreasing on $[0, \pi]$ so by a well-known theorem of Hardy [12, p 94] we have

$$
\int_{0}^{\pi}[(1+\cos ) \cdot f]^{\#} \Phi_{r}\left(g^{\#}\right) P_{r} d s \leq \int_{0}^{\pi}(1+\cos ) \cdot f^{\#} \Phi_{r}\left(g^{\sharp}\right) P_{r} d s .
$$

By symmetry, the same is true for the lower semicircle so these results combine to give

$$
\left\langle[(1+\cos ) \cdot f]^{\#}, \Phi_{r}\left(g^{\#}\right) P_{r}\right\rangle \leq\left\langle(1+\cos ) \cdot f^{\#}, \Phi_{r}\left(g^{\#}\right) P_{r}\right\rangle .
$$

Now this last estimate, together with Proposition 3.2, shows that

$$
\begin{aligned}
\left\langle\left(\Psi_{-r} f\right)^{\#}, g^{\#}\right\rangle & =\left\langle\left(\Phi_{-r}[1 / 2(1+\cos ) \cdot f]\right)^{\#}, g^{\#}\right\rangle \\
& \leq\left\langle\Phi_{-r}[1 / 2(1+\cos ) \cdot f]^{\#}, g^{\#}\right\rangle \\
& =\left\langle[1 / 2(1+\cos ) \cdot f]^{\#}, \Phi_{r}\left(g^{\#}\right) P_{r}\right\rangle \quad(\operatorname{cf} .(3.5)) \\
& \leq\left\langle 1 / 2(1+\cos ) \cdot f^{\#}, \Phi_{r}\left(g^{\#}\right) P_{r}\right\rangle=\left\langle\Psi_{-r}\left(f^{\#}\right), g^{\#}\right\rangle .
\end{aligned}
$$

Hence from (2.12),

$$
\begin{aligned}
\rho_{\mathfrak{X}}(-r) & =\sup _{\|f\|_{\mathfrak{X}} \leq 1}\left\|\Psi_{-r} f\right\|_{\mathfrak{X}}=\sup _{\|f\|_{\mathfrak{X}} \leq 1} \sup _{g \|_{\mathfrak{X}^{\prime} \leq 1}}\left\langle\left(\Psi_{-r} f\right)^{\#}, g^{\sharp}\right\rangle \\
& \leq \sup _{\|f\|_{\mathfrak{X}} \leq 1} \sup _{g \|_{X^{\prime} \leq 1}}\left\langle\Psi \Psi_{-r}\left(f^{\#}\right), g^{\#}\right\rangle .
\end{aligned}
$$


To obtain the reverse inequality, we note by (2.13) that the right -hand side of (5.6) does not exceed $\rho_{\mathfrak{X}}(-r)$ because $\left\|f^{\#}\right\|_{\mathfrak{X}}=\|f\|_{\mathfrak{X}}$ and $\left\|g^{\#}\right\|_{\mathfrak{X}^{\prime}}=\|g\|_{\mathfrak{X}^{\prime}}$.

Proposition 5.3. The nth iterate of $A_{\gamma}$ is given by

$$
\left(A_{\gamma}^{n} f\right)(t)= \begin{cases}(\tan t / 2)^{-\gamma} \int_{0}^{t}(\tan s / 2)^{\gamma-1} \frac{1}{(n-1) !}\left(2 \log \left(\frac{\sin t / 2}{\sin s / 2}\right)\right)^{n-1} f(s) d s, & 0<t<\pi / 2, \\ 0, \quad \pi / 2<t<2 \pi\end{cases}
$$

Proof. The proof is by induction on $n$ and is straightforward so we omit it. See [5, Lemma 2] for a similar argument.

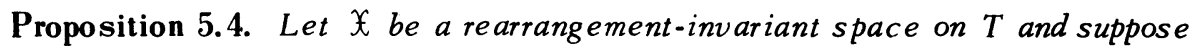
that $A_{\gamma}$ is a bounded operator on $\mathfrak{X}$ for some $\gamma, 0<\gamma \leq 1$. Then, for all sufficiently small $\epsilon>0, A_{\gamma-\epsilon}$ is a bounded operator on $\mathfrak{X}$.

Proof. (Cf. [5, pp. 22-24].) Choose any real number $\lambda>0$ such that $1 / \lambda$ exceeds the spectral radius of $A_{\gamma}$. Then [13, Chapter 1] $\left(I-\lambda A_{\gamma}\right)$ is invertible and

$$
\left(I-\lambda A_{\gamma}\right)^{-1}=\sum_{n=0}^{\infty} \lambda^{n} A_{\gamma}^{n} .
$$

Thus, if $f \in \mathfrak{X}$, we have from (5.7), for $0<t<\pi / 2$,

$$
\begin{aligned}
A_{\gamma}\left(I-\lambda A_{\gamma}\right)^{-1} f(t)=\sum_{n=0}^{\infty} \lambda^{n}\left(A_{\gamma}^{n+1} f\right)(t) \\
\quad=\sum_{n=0}^{\infty} \lambda^{n}(\tan t / 2)^{-\gamma} \int_{0}^{t}(\tan s / 2)^{\gamma-1} \frac{1}{n !}\left(2 \log \frac{\sin t / 2}{\sin s / 2}\right)^{n} f(s) d s \\
=(\tan t / 2)^{-\gamma} \int_{0}^{t}(\tan s / 2)^{\gamma-1}\left(\frac{\sin t / 2}{\sin s / 2}\right)^{2 \lambda} f(s) d s \\
=(\tan t / 2)^{2 \lambda-\gamma}(\cos t / 2)^{2 \lambda} \int_{0}^{t}(\tan s / 2)^{\gamma-2 \lambda-1}(\cos s / 2)^{-2 \lambda} f(s) d s .
\end{aligned}
$$

We further restrict the choice of $\lambda$ by here requiring that $2 \lambda<\gamma$. Let us now estimate the operator norm $\left\|A_{\gamma-2 \lambda}\right\|_{\boldsymbol{B}(\mathfrak{X})}$ of $A_{\gamma-2 \lambda}$ on $\mathfrak{X}$. Since $\left|A_{\gamma-2 \lambda}(f)\right| \leq$ $A_{\gamma-2 \lambda}(|f|)$ we need only consider nonnegative functions $f \in X$. Using (5.7) and the fact that $\cos t / 2 \geq 1 / 2$ on $[0, \pi / 2]$ we deduce from (5.8) that

$$
\begin{aligned}
\left(A_{\gamma-2 \lambda} f\right)(t) & =(\tan t / 2)^{\gamma-2 \lambda} \int_{0}^{t}(\tan s / 2 .)^{\gamma-2 \lambda-1} f(s) d s \\
& \leq 2 A_{\gamma}\left(I-\lambda A_{\gamma}\right)^{-1} f(t), \quad 0<t<\pi / 2 .
\end{aligned}
$$


But $A_{\gamma}$ and $\left(I-\lambda A_{\gamma}\right)^{-1}$ are bounded operators on $X$ so

$$
\left\|A_{\gamma-2 \lambda} f\right\|_{\mathfrak{X}} \leq 2\left\|A_{\gamma}\right\|_{\mathfrak{B}(\mathfrak{X})}\left\|\left(I-\lambda A_{\gamma}\right)^{-1}\right\|_{\mathfrak{B}(\mathfrak{X})}\|f\|_{\mathfrak{X}}
$$

and it follows that

$$
\left\|A_{\gamma-2 \lambda}\right\|_{\boldsymbol{B}(\boldsymbol{x})} \leq 2\left\|A_{\gamma}\right\|_{\boldsymbol{B}(\boldsymbol{x})}\left\|\left(I-\lambda A_{\gamma}\right)^{-1}\right\|_{\mathbb{B}(\mathfrak{x})}<\infty .
$$

This completes the proof.

Proposition 5.5. Let $\mathfrak{X}$ be a rearrangement-invariant space on $T$. Then if $0 \leq r<1,0<\gamma \leq 1$,

$$
\rho_{\mathfrak{X}}(-r) \leq \frac{\gamma}{2}\left(\frac{1-r}{1+r}\right)^{-\gamma}\left\|A_{\gamma}\right\|_{\boldsymbol{B}(\mathfrak{X})} .
$$

Proof. From (5.4) we have, for $0<t<\pi / 2$,

$$
\left(A_{\gamma} f\right)(t)=\int_{0}^{1} \frac{4}{(1+r)^{2}}\left(\frac{1-r}{1+r}\right)^{\gamma-1}\left(\Psi_{-r} f\right)(t) d r
$$

which, under the change of variable $s=(1-r) /(1+r)$, becomes

$$
\left(A_{\gamma} f\right)(t)=\int_{0}^{1} 2 s^{\gamma-1}\left(\Psi_{-s}^{\prime} f\right)(t) d s, \quad 0<t<\pi / 2,
$$

where $\Psi_{-s}^{\prime}=\Psi_{-r}, s=(1-r) /(1+r)$. Now if $f \in \mathfrak{X}, g \in X^{\prime},\left\langle\Psi_{-s}^{\prime}\left(f^{\sharp}\right), g^{\sharp}\right\rangle$ is decreasing in $s$ so

$$
\begin{aligned}
\left\langle\Psi_{-s}^{\prime}\left(f^{\#}\right), g^{\#}\right\rangle & \int_{0}^{s} 2 t^{\gamma-1} d t \leq \int_{0}^{s} 2 t^{\gamma-1}\left\langle\Psi_{-t}^{\prime}\left(f^{\#}\right), g^{\#}\right\rangle d t \\
& \leq \int_{0}^{1} 2 t^{\gamma-1} \frac{1}{2 \pi} \int_{0}^{2 \pi}\left(\Psi_{-t}^{\prime} f^{\#}\right)(\theta) g^{\#}(\theta) d \theta d t \\
& =\frac{1}{2 \pi} \int_{0}^{2 \pi} g^{\#}(\theta) d \theta \int_{0}^{1} 2 t^{\gamma-1}\left(\Psi_{-t}^{\prime} f^{\#}\right)(\theta) d t
\end{aligned}
$$

by Fubini's theorem. Hence from (5.10) and (2.13)

$$
\begin{aligned}
\left\langle\Psi_{-s}^{\prime}\left(f^{\#}\right), g^{\#}\right\rangle \frac{2}{\gamma} s^{\gamma} & =\frac{1}{2 \pi} \int_{0}^{2 \pi}\left(A_{\gamma}\left(f^{\#}\right)\right)(\theta) g^{\#}(\theta) d \theta \\
& =\left\langle A_{\gamma}\left(f^{\#}\right), g^{\#}\right\rangle \leq\left\|A_{\gamma}\right\|_{\mathfrak{B}(\mathfrak{X})}\|f\|_{\mathfrak{X}}\|g\|_{\mathfrak{X}} \cdot .
\end{aligned}
$$

Taking suprema, first over all $g \in X^{\prime}$ with $\|g\|_{X^{\prime}} \leq 1$ and then over all $f \in X$ with $\|f\|_{\mathfrak{x}} \leq 1$, we deduce from Lemma 5.2 that $\rho_{\mathfrak{X}}(-r) 2 / \gamma((1-r) /(1+r))^{\gamma} \leq$ $\left\|A_{\gamma}\right\|_{\mathcal{B}(x)}$. Thus (5.9) is established and the proof is complete.

Theorem 5.6. Let $\mathfrak{X}$ be a rearrangement-invariant space on $T$ with upper index $\xi<1$ and suppose that $0<y \leq 1$. Then $A_{\gamma}$ is a bounded operator on $\mathfrak{X}$ if and only if $\gamma>\xi$.

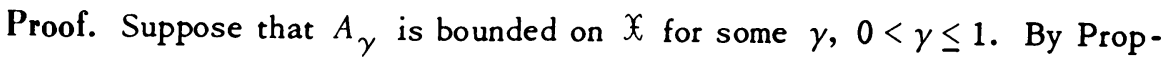
osition 5.4 , there is a number $\epsilon>0$ such that $A_{\gamma-\epsilon}$ also is bounded on $X_{\text {. But }}$ then by Proposition 5.5, 


$$
\rho_{\mathfrak{X}}(-r) \leq \frac{\gamma-\epsilon}{2}\left(\frac{1-r}{1+r}\right)^{\epsilon-\gamma}\left\|A_{\gamma-\epsilon}\right\|_{\mathfrak{B}(\mathfrak{X})} \leq \mathrm{const}\left(\frac{1-r}{1+r}\right)^{\epsilon-\gamma}
$$

so we deduce from (4.14) that

$$
\xi=\lim _{r \rightarrow 1} \frac{\log \rho_{x}(-r)}{\log ((1+r) /(1-r))} \leq \gamma-\epsilon<\gamma,
$$

as required. The converse result was established in Theorem 5.1 so the proof of Theorem 5.6 is complete.

6. An interpolation theorem. Let $\tau$ be the mapping of $[0,2 \pi]$ onto $T$ defined by $r(\theta)=e^{i \theta}, 0 \leq \theta \leq 2 \pi$. When $X$ is a rearrangement-invariant space on $T$, the space $\mathfrak{X}_{0}$ consisting of all functions $F=f \circ \tau, f \in \mathfrak{X}$, is a rearrangement-invariant space on $[0,2 \pi]$ under the norm

$$
\|F\|_{\mathfrak{x}_{0}}=\|f\|_{\mathfrak{x}}, \quad F=f \circ \tau, f \in \mathfrak{X} .
$$

As in [6], define the averaging operator $P_{\gamma}, 0<\gamma \leq 1$, by

$$
\left(P_{\gamma} F\right)(t)=t^{-\gamma} \int_{0}^{t} s^{\gamma-1} F(s) d s, \quad 0<t \leq 2 \pi .
$$

Theorem 6.1. Let $\mathfrak{X}$ be a rearrangement-invariant space on $T$ and suppose

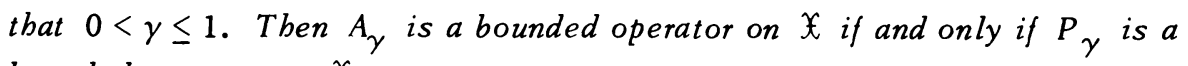
bounded operator on $\mathfrak{X}_{0}$.

Proof. Comparing the definitions (5.1), (6.2) of $A_{\gamma}$ and $P_{\gamma}$ we see that the basic difference is that $\tan t / 2$ and $t$ are interchanged. However, since $t^{-1} \tan t / 2$ and $t(\tan t / 2)^{-1}$ are both bounded functions on [0, $\left.\pi / 2\right]$, it is clear that $P_{\gamma}$ bounded on $\mathfrak{X}_{0}$ implies $A_{\gamma}$ bounded on $\mathfrak{X}$.

For the converse, we have to take into account the fact that $A_{\gamma}$ is defined to be zero on $[\pi / 2,2 \pi]$ whereas $P_{\gamma}$ is not.

Now, if $\pi / 2<t<2 \pi$ and $F \geq 0$,

$$
\begin{aligned}
\left(P_{\gamma} F\right)(t) & =t^{-\gamma} \int_{0}^{\pi / 2} s^{\gamma-1} F(s) d s+t^{-\gamma} \int_{\pi / 2}^{t} s^{\gamma-1} F(s) d s \\
& \leq \text { const }\left(A_{\gamma} f\right)\left(\frac{\pi}{2}\right)+\frac{2}{\pi} \int_{0}^{2 \pi} F(s) d s, \quad F=f \circ \tau .
\end{aligned}
$$

The integral $\int_{0}^{2 \pi} F(s) d s$ converges because $\mathfrak{X}_{0} \subset L^{1}[0,2 \pi]$ (cf. $\S 2$ ), so (6.3) shows that $P_{\gamma} F$ is bounded on $[\pi / 2,2 \pi]$. But $L^{\infty}[0,2 \pi] \subset \mathfrak{X}_{0}$ (cf. $§ 2$ ) so this implies that

$$
\left(P_{\gamma} F\right) \chi_{1} \in X_{0}, \quad F \in X_{0},
$$

where $\chi_{1}$ is the characteristic function of $[\pi / 2,2 \pi]$.

On the other hand, if $\chi_{0}$ is the characteristic function of $[0, \pi / 2]$, we proceed as in the first half of the proof to find that 
in particular,

$$
\left\|\left(P_{\gamma} F\right) \chi_{0}\right\|_{\mathfrak{X}_{0}} \leq \text { const }\left\|A_{\gamma}\right\|\|f\|_{\mathfrak{X}}=\text { const }\|F\|_{\mathfrak{X}_{0}}<\infty
$$

$$
\left(P_{\gamma} F\right)_{\chi_{0}} \in \mathfrak{X}_{0}, \quad F \in \mathfrak{X}_{0} .
$$

Taking (6.4) and (6.5) together we deduce that $P_{\gamma}: X_{0} \rightarrow X_{0}^{\prime}$.

It remains only to show that $P_{\gamma}$ is bounded. If not then we can find functions $F_{n} \in X_{0}, n=1,2, \cdots$, with $\left\|F_{n}\right\|_{\mathfrak{X}_{0}} \leq 2^{-n}$ such that $\left\|P_{\gamma} F_{n}\right\|_{\mathfrak{X}_{0}}>n$. But $\mathfrak{X}_{0}$ is complete so $F=\sum_{n=1}^{\infty} F_{n} \in X_{0}$; moreover, $F_{n} \leq F$ implies $P_{\gamma} F_{n} \leq P_{\gamma} F$ and so $\left\|P_{\gamma} F\right\|_{\mathfrak{x}_{0}} \geq\left\|P_{\gamma} F_{n}\right\|>n, n=1,2, \cdots$. Hence $P_{\gamma} F \notin X_{0}$ which is a contradiction because $F \in \mathfrak{X}_{0}$ and $P_{\gamma}: \mathfrak{X}_{0} \rightarrow \mathfrak{X}_{0}$. Therefore, $P_{\gamma}$ is bounded on $\mathfrak{X}_{0}$ and the proof of Theorem 6.1 is complete.

We refer the reader to [6] for the definition of the Boyd indices $\alpha, \beta$. An immediate consequence of the definition is that $\alpha(X)=\alpha\left(X_{0}\right)$ and $\beta(X)=\beta\left(X_{0}\right)$.

Theorem 6.2. Let $\mathfrak{X}$ be a rearrangement-invariant space on $T$ with indices $\xi, \eta$. Let $\alpha, \beta$ denote the Boyd indices of $\chi$. Then $\xi=a$ and $\eta=\beta$.

Proof. Fix $\xi$ with $0 \leq \xi<1$ and suppose $\gamma$ satisfies $0<\gamma \leq 1$. By Theorem 5.6, $\gamma>\xi$ if and only if $A_{\gamma}$ is bounded on $\mathfrak{X}$. But by Theorem 6.1, this is equivalent to the boundedness of $P_{\gamma}$ on $\mathfrak{X}_{0}$ which in turn occurs if and only if $\gamma>a[6$, p. 1253]. This shows that if $\xi<1$, then $\xi=a$.

Now a similar argument shows that if $a<1$, then $a=\xi$. It follows that $0 \leq \alpha<1$ if and only if $0 \leq \xi<1$ (and in this case $\alpha=\xi$ ). But this surely implies that $\dot{\alpha}=1$ if and only if $\xi=1$. Hence, whatever the values of $\xi$ and $a$ we have always $\xi=\alpha$.

Applying this result to the associate space $X^{\prime}$ we find $\xi^{\prime}=\alpha^{\prime}$ and hence, by (4.17) and [6, Lemma 5], $\eta=1-\xi^{\prime}=1-\alpha^{\prime}=\beta$.

Theorem 6.2 and [6, Theorem 1] can now be combined to produce the following interpolation theorem (a special case was established in [1] using different methods).

Theorem 6.3. Let $\mathfrak{X}$ be a rearrangement-invariant space on $T$ with indices $\xi, \eta$. Then every linear operator of weak types $(p, p)$ and $(q, q)$ is a bounded operator on $\mathfrak{X}$ if and only if $p^{-1}>\xi$ and $q^{-1}<\eta$.

\section{REFERENCES}

1. C. Bennett, On the harmonic analysis of rearrangement-invariant Banach function spaces, Thesis, University of Newcastle, 1971.

2. C. Bennett and J. E. Gilbert, Homogeneous algebras on the circle. I. Ideals of analytic functions, Ann. Inst. Fourier (Grenoble) 22 (1972).

3. - Homogeneous algebras on the circle. II. Multipliers, Ditkin conditions, Ann. Inst. Fourier (Grenoble) 22 (1972). 
4. D. W. Boyd, The Hilbert transform on rearrangement-invariant spaces, Canad. J. Math. 19 (1967), 599-616. MR $35 \# 3383$.

5. - The spectral radius of averaging operators, Pacific J. Math. 24 (1968), 19-28. MR $36 \# 4360$.

6. - Indices of function spaces and their relationship to interpolation, Canad. J. Math. 21 (1969), 1245-1254.

7. A. P. Calderón, Spaces between $L^{1}$ and $L^{\infty}$ and the theorem of Marcinkiewicz, Studia Math. 26 (1966), 273-299. MR $34 \# 3295$.

8. E. Hille and R. S. Phillips, Functional analys is and semi-groups, rev. ed., Amer. Math. Soc. Colloq. Publ., vol. 31, Amer. Math. Soc., Providence, R. I., 1957. MR 19, 664.

9. G. G. Lorentz and T. Shimogaki, Interpolation theorems for operators in function spaces, J. Functional Analysis 2 (1968), 31-51. MR $41 \# 2424$.

10. - Interpolation theorems for the pairs of spaces $\left(L^{p}, L^{\infty}\right)$ and $\left(L^{1}, L^{q}\right)$, Trans. Amer. Math. Soc. 159 (1971), 207-221.

11. W. A. J. Luxemburg, Banach function spaces, Thesis, Technische Hogeschool te Delft, 1955. MR 17, 285.

12. - Rearrangement-invariant Banach function spaces, Queen's Papers in Pure and Appl. Math., no. 10, Queen's University, Kingston, Ont., 1967, pp. 83-144.

13. C. E. Rickart, General theory of Banach algebras, University Series in Higher Math., Van Nostrand, Princeton, N. J., 1960. MR 22 \#5903.

14. T. Shimogaki, An interpolation theorem on Banach function spaces, Studia Math. 31 (1968), 233-240. MR $38 \# 2618$.

DEPARTMENT OF MATHEMATICS, CALIFORNIA INSTITUTE OF TECHNOLOGY, PASADENA, CALIFORNIA 91109 